\title{
La lutte contre l'East Coast Fever par bains détiqueurs au Malawi : le programme du point de vue de l'éleveur
}

\author{
F. Blanc ${ }^{1 *}$, T. Norman $^{2}$, A. Soldan ${ }^{2}$, D. Chilombo ${ }^{2}$, M. Edelsten ${ }^{2}$
}

BLANC F, YORMAN T., SOLDAN A., CHILOMBO D., EDELSTEN M. La lutte contre l'East Coast Fever par bains détiqueurs au Malawi : le programme du point de vue de l'éleveur. Revue Elev. Méd. vét. Pays trop., 1995, 48 (4) : 307-314

La lutte par bains détiqueurs contre l'East Coast Fever (ECF) au Malawi entraîne une réduction des pertes dues à cette maladie variable avec la fréquence de balnéation. Le seul coût pour l'éleveur est représenté par le temps passé à la manipulation des animaux. Toutefois, l'impact économique de l'ECF apparaît limité en situation d'élevage traditionnel. Les éleveurs tiennent son importance comme secondaire par rapport à d'autres interventions en santé ou production animales ou dans d'autres domaines de leur systène de production. Les caractéristiques de ces systèmes expliquent les différences d'intérêt pour les bains entre les divers types d'éleveurs. Vus les con̂ts élevés de cette méthode de lutte, diverses modifications sont envisagées.

Mots-clés : Theilériose - Tique - Immersion - Lutte anti-acarien - Contrôle de maladies - Coût - Malawi.

\section{INTRODUCTION}

Les programmes intensifs de bains détiqueurs pour le contrôle des maladies à tiques, notamment de la plus virulente, l'East Coast Fever (ECF), semblent actuellement remis en question en situation d'élevage traditionnel. Ces programmes sont extrêmement coûteux pour les services vétérinaires à un moment où les devises se font chères. Ils peuvent conduire à des catastrophes pour l'élevage, comme cela s'est produit au Zimbabwe, en perturbant les stabilités enzootiques qui se créent entre le bétail et les agents pathogènes (10). Enfin, du fait de la méconnaissance du coût économique réel de ces maladies sur le bétail local, le bénéfice de ces programmes reste souvent mal évalué. II semble toutefois que ces mesures intensives n'entraînent pas en élevage traditionnel les bénéfices qui justifieraient leur coût.

Cet article étudie le point de vue de l'éleveur vis-àvis de la lutte par bains détiqueurs du Malawi. Trois questions y sont posées : i) cette technique de lutte présente-t-elle des contraintes variables selon les caractéristiques

1. Centre for Tropical Veterinary Medicine (CTVM), Easter Bush, Roslin, EH25 9RG, Edinburgh, Royaume-Uni.

2. Livestock Disease Evaluation Unit (LDEU), Central Veterinary Laboratory, P.O. Box 527, Lilongwe, Malawi.

* Adresse actuelle: NNRDP, PO Box 498, Oshakati, Namibie.

Reçu le 4.3.94, accepté le 7.2.96. socio-économiques des types d'élevage ? ii) quels sont les coûts et les bénéfices des balnéations pour les éleveurs ? iii) les éleveurs peuvent-ils participer financièrement à une lutte dont la poursuite est compromise par les frais élevés de fonctionnement?

Considérer le point de vue de l'éleveur dans un tel programme présente plusieurs intérêts : i) cette approche permet d'identifier la place du bétail dans le système de production agricole, et de savoir comment une intervention technique s'y inscrit, pour une meilleure connaissance de l'impact de cette intervention ; ii) connaître quels sont les intérêts et les contraintes d'un programme pour les éleveurs permet une adaptation souple aux situations rencontrées ; iii) ces informations constituent une aide à la décision pour les décideurs. Les mesures pourront être négociées et donc plus facilement acceptées que si elles étaient imposées; iv) une telle étude est indispensable pour définir un optimum socio-économique au sens de Pareto ou de Hicks-Kaldor (15).

\section{Le Malawi, l'élevage, l'East Coast Fever...}

Le Malawi est un pays d'Afrique de l'Est avec une forte densité de population. Celle-ci est essentiellement agricole $(85 \mathrm{p}$. 100) et les petites exploitations sont prédominantes (90 p. 100) (7). Le cheptel national est de 800000 têtes. Ce sont à 95 p. 100 des zébus de type Sanga, les Malawi local zebu, élevés en milieu traditionnel par petits troupeaux (médiane de 7,3 animaux par troupeau (2)). Cette petite race locale, dont l'aspect s'apparente plus à celui d'un N'Dama qu'à celui d'un zébu Mbororo, peut être supposée adaptée à son milieu et "résistante". La présente étude ne concerne que cette race locale et sa situation vis-à-vis de l'ECF, à l'exclusion de tout système amélioré.

L'ECF est présente dans le Nord et le Centre du Malawi (figure 1 , d'après Daborn (5)) où son vecteur, l'espèce de tique Rhipicephalus appendiculatus, se rencontre (1). Cette maladie a longtemps été considérée comme la dominante pathologique en élevage bovin $(11,21)$. Un programme obligatoire de bains hebdomadaires a été institué afin de l'éradiquer, objectif qui paraît désormais hors d'atteinte.

L'opinion selon laquelle l'ECF infligeait des pertes considérables au bétail a été révisée en situation d'élevage traditionnel. L'impact de l'ECF s'est trouvé considérable- 


\section{F. Blanc T. Norman A. Soldan D. Chilombo M. Edelsten}

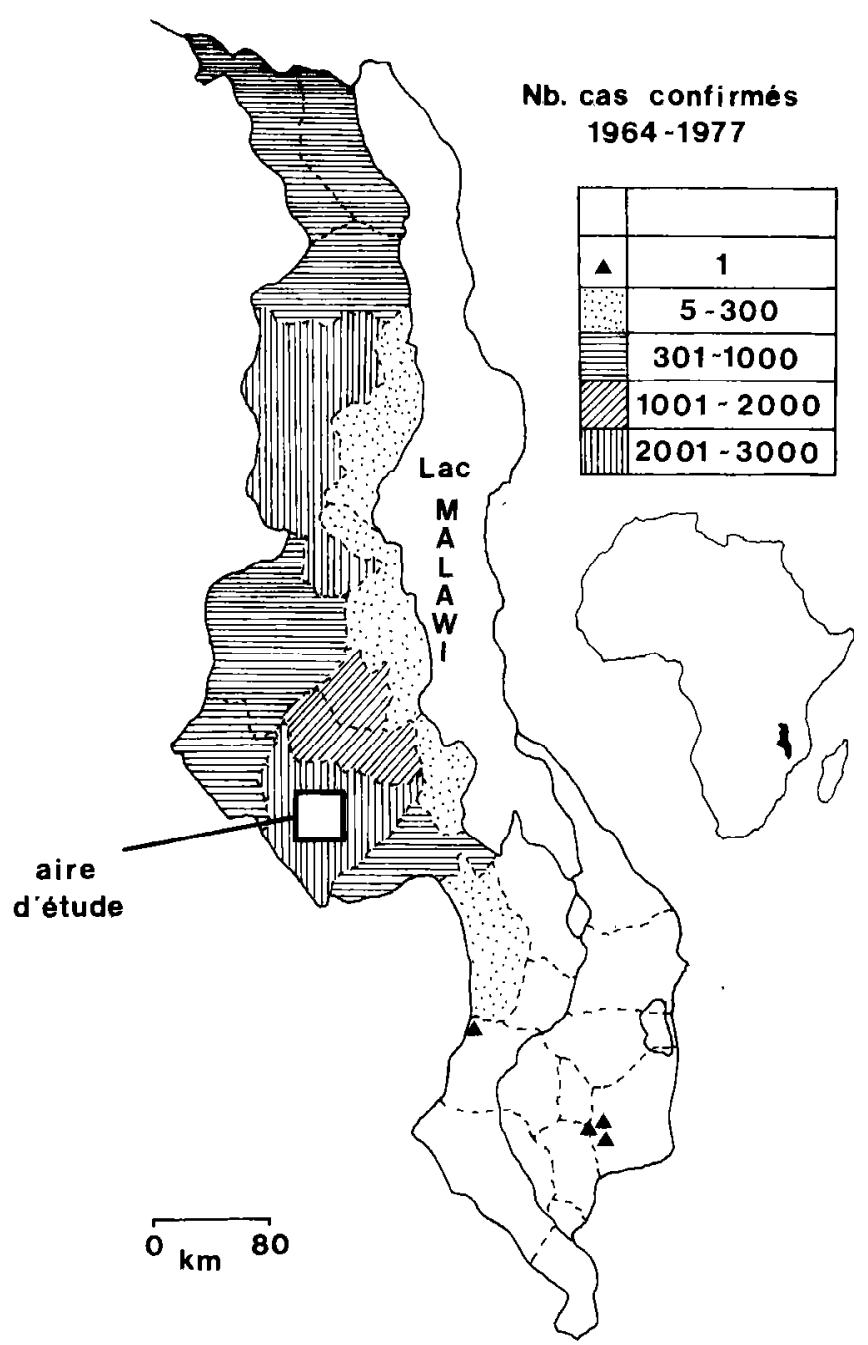

Figure 1: Distribution de l'ECF au Malawi, d'après Daborn (1981) (5).

ment réduit par différentes études qui par contre insistaient sur le coût des balnéations $(9,17,22)$. Progressivement ces coûts sont apparus supérieurs aux bénéfices de production dus à la balnéation (8).

\section{MATERIEL et METHODES}

\section{Trois sources ont été utilisées :}

i) une enquête a été menée dans huit aires d'élevage autour de Lilongwe, dans la région la plus exposée à l'ECF avec le district de Mzuzu (5). Cent cinq éleveurs, possédant un total de 1006 animaux, ont été consultés (figure 1);

ii) les registres tenus par l'assistant vétérinaire responsable du bain détiqueur de Tanga, à $20 \mathrm{~km}$ au sud de
Lilongwe, ont été analysés. Le fonctionnement de ce bain avait été satisfaisant dans les années passées ;

iii) les résultats du suivi d'un réseau d'élevages $(13,18)$ regroupant 1800 animaux marqués, mené par le Livestock Disease Evaluation Unit (LDEU) dans la même région depuis deux ans, ont servi à l'évaluation des pertes dues à l'ECF. Ces pertes, notamment les taux de morbidité et de mortalité pour différentes fréquences de balnéation, utilisées pour l'évaluation économique, ne pouvaient pas être évaluées aussi précisément au cours d'une enquête rapide.

Le point de vue de l'éleveur devait être défini à la fois dans ses aspects économiques et sociaux. Pour ces derniers, une analyse factorielle des correspondances (AFC) (6) a été utilisée pour définir les caractéristiques de groupes d'éleveurs selon leur degré de motivation envers la balnéation.

La valeur du bétail pour l'éleveur ne se limite pas à son prix de boucherie. De même le coût des mortalités ne se résume pas à la perte du prix à la vente de l'animal. D'abord une partie de la viande est récupérée et consommée ; ensuite l'animal disparu représente une perte de production (d'un veau, de fumier, etc.). Une nouvelle valeur du bétail tenant compte de ces remarques a été établie* à partir des résultats de l'enquête : la taille nationale du cheptel restant constante depuis plusieurs années, toute réduction des pertes se traduit par un surcroît d'exploitation à court terme.

Le prix de marché d'un adulte peut être considéré comme représentatif à la fois de sa qualité bouchère et de ses potentialités productives pour l'éleveur. Par contre la valeur des jeunes utilisée tient compte du prix qu'ils auront à l'age adulte, diminué de la probabilité de ne pas atteindre cet age adulte". C'est une sorte de "valeur potentielle": Les coûts moyens d'élevage (50 MK"to par animal par an) sont déduits et la valeur des produits (fumier, viande récupérée en cas d'abattage d'urgence, prix obtenu à la vente) rajoutés.

Cette valeur est utilisée pour calculer le coût de mortalité d'un animal jeune. La perte de production de fumier est rajoutée à ce coût, et la part de viande récupérée (en général consommée) déduite. Les pertes de fumier, d'utilisation pour la traction (pour les mâles), d'un veau et d'une lactation (pour les femelles) sont ajoutées à la valeur bouchère pour la mortalité des adultes. La part de viande récupérée est déduite.

\footnotetext{
* Ces bénéfices et ces coûts de l'activité de la production animale ne seront pas détaillés ici ; pour plus de détails, se rérérer au rapport dont cet article s'inspire (2), qui peut être obtenu sur demande aux auteurs.

** C'est-à-dire 100 p. 100 - les taux de mortalité, de vente et de consommation.

** 1 Malawi Kwacha $(\mathrm{MK})=100$ tambala $=1,50 \mathrm{FF}$
} 


\section{RESULTATS}

\section{La valeur du bétail et le coût des mortalités}

Le tableau I présente les valeurs établies et le tableau II le coût pour l'éleveur d'une mortalité, selon l'âge et le sexe des animaux.

\section{Les déclarations des éleveurs}

L'ECF représente 24 p. 100 des mortalités totales avec une fréquentation moyenne des bains entre 50 et 60 p 100 sur la zone*. Ces mortalités totales restent raisonnables : 12,5 p. 100 pour les veaux entre 0 et 1 an; 4,2 p. 100 pour les animaux entre 1 et 3 ans ; 5 p. 100 pour les adultes. Les pertes par "négligence" d'entretien (veaux noyés dans le parc en saison des pluies, blessures mortelles au pâturage), qui seraient beaucoup plus faciles à prévenir, représentent également 24 p. 100 des mortalités.

TABLEAU I

Valeur des animaux utilisée dans les calculs (en MK). Moyenne pondérée pour chaque classe en tenant compte du sex ratio

\section{Femelles}

Mâles Moyenne

Valeur d'un animal 0 à 1 an 353 $461 \quad 397$

Valeur d'un animal 1 à 3 ans 448

$650 \quad 512$

Valeur d'un animal > 3 ans" 500 $750 \quad 583$

$1 \mathrm{MK}:$ Malawi Kwacha $=100$ tambala $=1,50 \mathrm{FF}$.

\section{TABLEAU II}

Calcul des pertes entraînées par une mortalité (en MK), en reprenant les résultats de Norman et Soldan $(1992)(13,18)$, et les productions du bétail établies lors de l'enquête

\section{Types d'animaux}

Mâle - femelle 0 à 1 an

Mâle - femello 1 à 3 ans

\section{Mâle > 3 ans}

Femelle $>3$ ans

Moyenne pour animaux $>3$ ans.

.

- $66,6 \%$ sont des femelles et $33,3 \%$ des mâles

\footnotetext{
* Les renseignements obtenus sont obscurcis et biaisés par l'obligation de la balnéation hebdomadaire.
}

\section{Pertes (MK)}

350

409

634

475

528

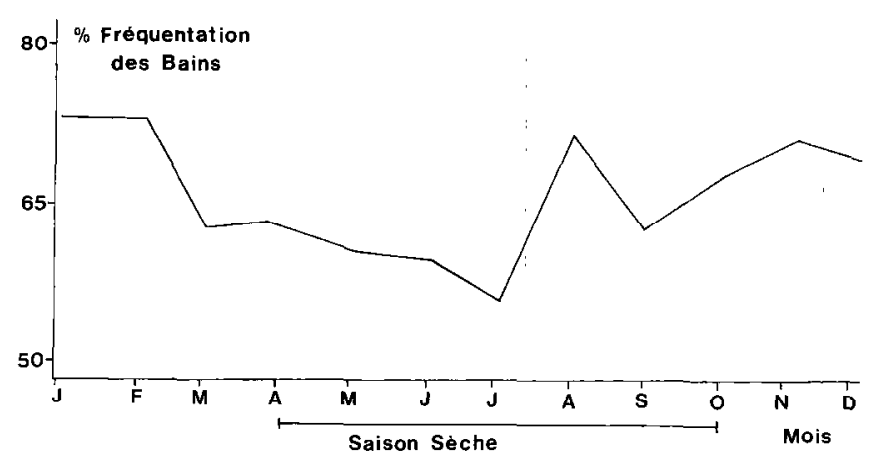

Figure $2:$ Fréquentation du bain détiqueur selon la saison.

Une fréquentation de 100 p. 100 signifie que tous les éleveurs viennent 52 fois par an au bain détiqueur. Elle passe à 50 p. 100 si seulement 50 p. 100 des éleveurs viennent 52 fois, ou si 100 p. 100 des éleveurs viennent 1 semaine sur 2 , etc.

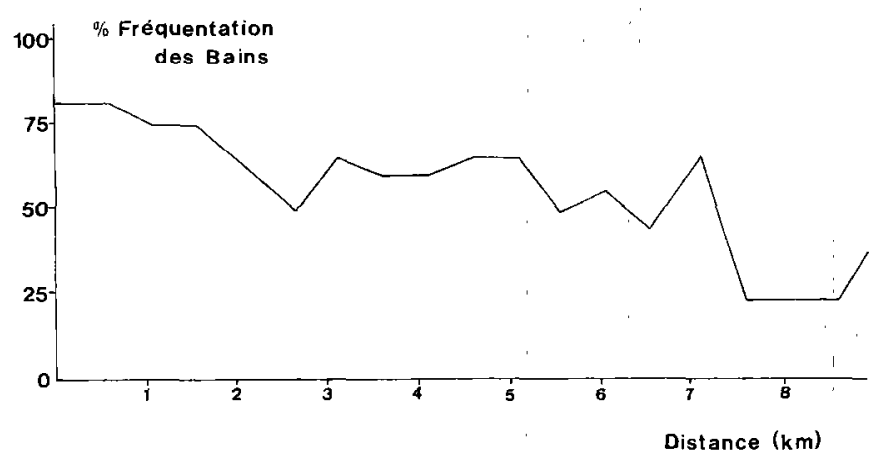

Figure 3 : Fréquentation selon la distance de la ferme au bain détiqueur.

L'amélioration des soins de santé occupe une place secondaire dans l'ordre des priorités citées par les éleveurs. Le contrôle des maladies à tiques n'est guère ressenti comme une priorité. Parmi les raisons fournies comme obstacle à la balnéation sont citées : i) la difficulté de se déplacer pendant la saison des pluies du fait de l'inondation de certaines zones; ii) la priorité donnée aux travaux des champs en cette saison; iii) l'absence de surveillance des animaux en saison sèche lorsque le bouvier a été congédié.

\section{Les registres du bain détiqueur}

La fréquentation du bain détiqueur est loin d'atteindre les 52 bains théoriques. Les facteurs influant leur fréquentation sont :

- la saison : c'est en saison des pluies que la fréquentation des bains est la plus élevée (figure 2);

- la distance au bain détiqueur : la fréquentation baisse lorsque cette distance augmente (figure 3 ), tendance déjà observée au Burundi (4); 


\section{F. Blanc T. Norman A. Soldan D. Chilombo M. Edelsten}

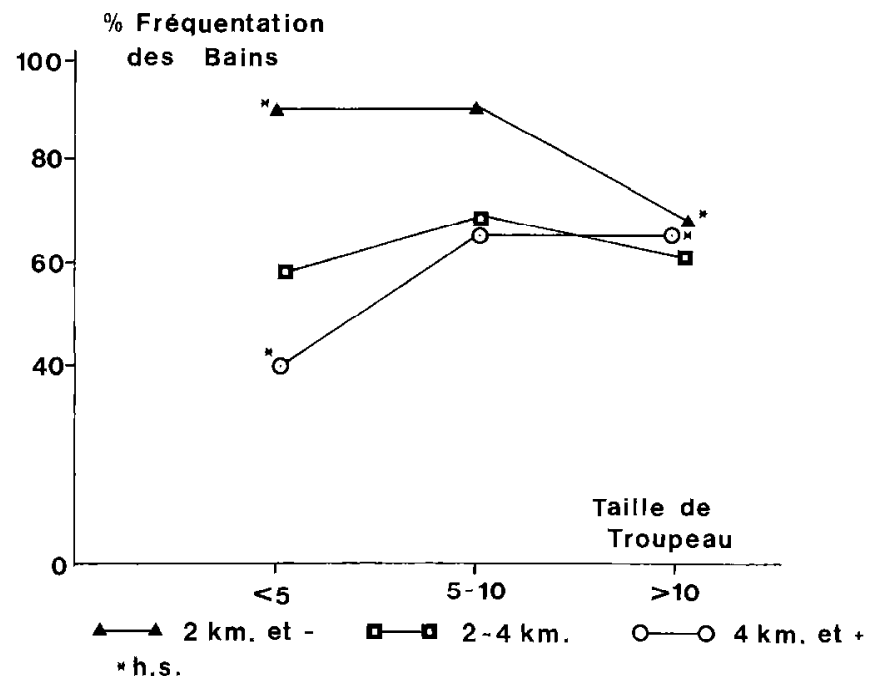

Figure 4 : Fréquentation selon la taille du troupeau. h.s.: différence de fréquentation selon la distance hautement significative pour une même taille de troupeau.

- la taille du troupeau : ce facteur devait être étudié en supprimant l'effet de la distance (figure 4) ; lorsque la distance n'est pas une contrainte (moins de $2 \mathrm{~km}$ ) les petits troupeaux sont baignés plus souvent que les grands troupeaux. Pour les distances éloignées (plus de $4 \mathrm{~km}$ ) c'est l'inverse : la fréquentation augmente lorsque la taille du troupeau augmente.

\section{L'analyse factorielle des correspondances : le profil social de la motivation des éleveurs}

Les caractéristiques des éleveurs favorables ou défavorables à la balnéation, obtenues à la suite des projections de l'AFC, sont résumées dans le tableaù III.

\section{TABLEAU III}

Caractéristiques des éleveurs classés selon leur adhésion à la balnéation



TABLEAU IV

Bénéfices de la balnéation pour l'éleveur (en MK). Calcul pour trois fréquences de balnéation et 1000 animaux



\section{Evaluation économique du point de vue de l'éleveur}

Les seuls bénéfices de la balnéation sont une réduction des taux de morbidité et des taux de mortalité dues à l'ECF *. Ces pertes et, par déduction, les avantages de la balnéation (tableau IV) sont établis à l'aide des évaluations du tableau II, des taux de mortalité et des nombres de traitement (13) pour trois fréquences : 0,17 et 52 bains par an (en supposant que cette dernière assure une protection intégrale).

Le bénéfice brut que l'éleveur tire de la balnéation n'apparaît pas comme une fonction linéaire de la fréquence, du fait notamment de l'avantage supérieur de baigner en saison des pluies. Passé une certaine fréquence, le bénéfice marginal tiré d'un bain supplémentaire se réduit. La courbe semble être une asymptote (figure 5), avec un point d'inflexion situé entre 17 et 30 bains par an. Le bénéfice pour le troupeau, égal à la somme des bénéfices individuels, augmente proportionnellement à la taille du troupeau.

Le seul coût identifiable de la balnéation pour l'éleveur est le temps passé à conduire les animaux au bain déti-

* L'ECF, hormis deux cas de babésiose sur 1800 animaux (13, 18), est la seule maladie à tiques détectée. 


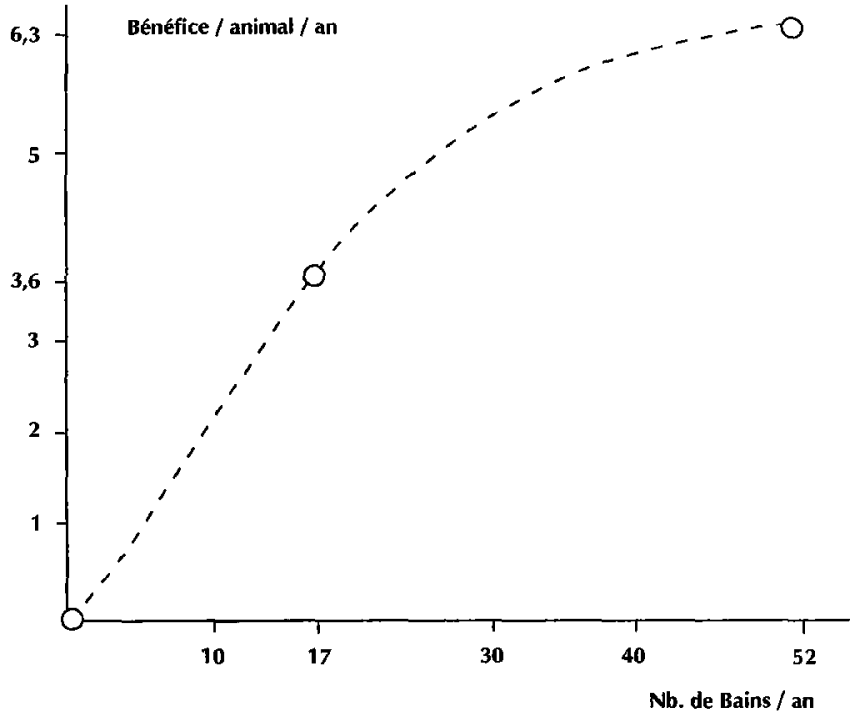

Figure 5 : Bénéfice brut de l'éleveur selon la fréquence de balnéation.

queur : certains éleveurs y accompagnent parfois le bouvier. Le taux de retour au travail agricole permet de chiffrer ce coût, en supposant que le temps passé sur le chemin du bain détiqueur est perdu pour le travail au champ. La valeur médiane du retour net au travail agricole est de 0,26 MK par heure. La taille du troupeau par contre n'influe pas sur ce coût, la manipulation étant identique quelle que soit cette taille.

Aucune différence d'état corporel n'a pu être mise en évidence entre animaux baignés et non baignés. Aucun coût ne peut donc être imputé, à l'inverse de l'étude de Nxumalo (14), à l'effort que représente le déplacement du bétail. L'alimentation, uniquement assurée par le pâturage, constitue une ressource sans frais.

Le temps de déplacement, et donc le coût pour l'éleveur, augmente en fonction de la distance au bain (régression significative, $\mathrm{r}^{2}=56 \%$, figure 6 ). Contrairement au bénéfice, ce coût augmente aussi linéairement en fonction de la fréquence de balnéation. Une telle constance des coûts au cours de l'année repose sur trois points : i) les éleveurs sont occupés dans les champs 10 mois sur 12 au moins; ii) en saison sèche d'autres opportunités de travail se présentent; iii) en saison sèche la manipulation des animaux incombe directement à l'éleveur.

\section{DISCUSSION}

\section{Les registres du bain détiqueur}

Les éleveurs sont conscients des avantages de baigner leur cheptel en saison des pluies, lorsque le risque d'ECF

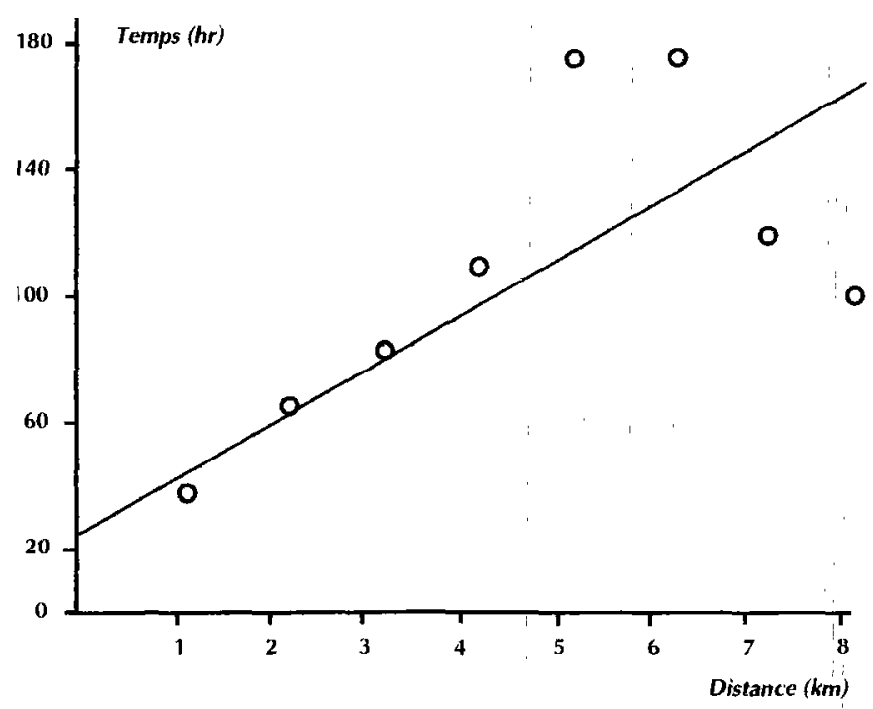

Figure 6 : Cồt de la balnéation pour l'éleveur selon la distance au bain détiqueur représenté pour une fréquence de 52 bains par an.

s'accroît du fait de la prolifération des tiques. Ils y répondent par une fréquentation plus assidue du bain détiqueur. Les travaux des champs les accaparent alors davantage, mais cette contrainte s'efface devant l'intérêt d'une meilleure protection.

Certaines hypothèses peuvent être émises :

- La balnéation présente des avantages connus des éleveurs. Le bénéfice qu'ils en tirent varie selon la taille des troupeaux et la période de l'année.

- Bien que la balnéation soit gratuite pour l'éleveur, elle n'est cependant pas "sans coût" ; ce coût augmente avec la distance au bain.

- Une fréquentation de 100 p. 100 sur une année ne représente pas pour l'éleveur l'optimum économique, qui doit être établi en tenant compte des coûts comme des bénéfices.

\section{Le profil social de la motivation des éleveurs}

D'après l'AFC, les principales caractéristiques des éleveurs favorables ou plutôt favorables à la balnéation sont :

- la proximité du bain, déjà expliquée ;

- la possession d'un petit troupeau, qui peut s'expliquer par une aversion pour le risque : l'éleveur tend à surprotéger le peu d'animaux qu'il possède et qui ont une utilité maximale dans son système de production; 
- l'absence d'utilisation du fumier pour les cultures, liée au fait que les petits éleveurs ont rarement des bœufs de traction pour le transport de ce fumier ;

- la possession de troupeaux importants, qui sera expliquée dans l'évaluation économique ;

- une attitude de fermier "progressiste", intéressé par les innovations techniques. La balnéation est vraisemblablement vue comme faisant partie d'un ensemble de "techniques améliorées".

A l'inverse les caractéristiques des éleveurs plutôt ou franchement défavorables à la balnéation sont :

- l'éloignement du bain détiqueur ;

- la possession des troupeaux les plus grands. Ces éleveurs, les plus aisés, sont impliqués dans diverses activités non-agricoles (épicerie, etc.) ou possèdent des "plantations" importantes. Leurs efforts se concentrent sur ces autres occupations plus rentables. Ils peuvent se permettre de "sacrifier" quelques têtes sans que l'utilité qu'ils tirent du bétail soit diminuée de manière conséquente par cette perle ;

- l'implication dans diverses activités non agricoles, liée au caractère précédent.

Ces éleveurs aisés sont probablement ceux qui disposent de la plus forte influence sociale et les plus à même de déterminer les mouvements d'opinion. Leur désintérêt pour la balnéation rend possible un changement de stratégie dans un sens moins favorable aux éleveurs, par exemple en faisant accepter plus facilement de faire payer le passage au bain : ces "faiseurs d'opinion" ne se mobiliseront pas ni ne mobiliseront les autres éleveurs contre ces changements. Par ailleurs les fermiers se concertent peu voire jamais entre eux sur les problèmes de leurs troupeaux. L'assistant vétérinaire responsable du bain détiqueur apparaît comme la source de toute information concernant l'élevage. Cette situation est favorable à une vulgarisation efficace à partir de ces assistants ; elle est défavorable à un mouvement d'opinion autonome et concerté des fermiers.

\section{Evaluation économique}

Le coût de l'ECF reste limité même en absence de toute mesure de lutte contre les tiques (fréquence annuelle de 0 ). Ceci explique le peu de priorité que donnent les éleveurs au programme de balnéation, et confirme l'importance réduite que cette maladie a sur le bétail traditionnel au Malawi. Une conclusion similaire avait été obtenue au Zaïre (3).

Figure 5, la relation bénéfices/fréquence de balnéation établie à l'aide de trois points seulement, reste toutefois une hypothèse. Elle suppose que les bains de saison des pluies, dont l'efficacité est maximale, sont effectués en priorité ; les bains suivants sont effectués avec des popu- lations de tiques de plus en plus réduites. Cette hypothèse ne tient pas compte d'une réduction accrue de la population de tiques si la proportion d'éleveurs appliquant le programme augmente. Elle n'envisage pas les perturbations qu'une balnéation intensive peut entraîner sur l'état de stabilité enzootique vis-à-vis des autres maladies à tiques. De plus ces données mesurées sur deux ans doivent être confirmées sur d'autres années lorsque les conditions climatiques varient.

Les résultats obtenus l'année suivante ont montré une incidence plus importante de l'ECF, des pertes en conséquence plus élevées et une amélioration très forte des effets d'une balnéation limitée à neuf bains annuels (19); ces résultats ne remettent en cause ni la méthode adoptée dans cette étude, ni les conclusions des deux premières années, mais ils soulignent l'importance de mener des études sur une période de temps suffisamment longue.

Le calcul de l'optimum économique de l'éleveur doit se faire en tenant compte de bénéfices dont l'accroissement, au-delà d'une certaine fréquence, est moins que proportionnel à l'augmentation des coûts. Le bénéfice net de l'éleveur dépend donc de la situation de chacun en fonction de la taille de son troupeau et de la distance au bain détiqueur. Chaque éleveur, s'il dispose du choix de sa propre fréquence, tendra à adopter une fréquence différente. De son point de vue, l'obligation d'un bain hebdomadaire n'est ni souple ni favorable, ce qui explique qu'elle soit peu suivie. La fréquence de 52 bains par an est de toute manière éloignée de cet optimum, qui se situe le plus souvent entre 9 et 30 bains selon la distance au bain, la taille du troupeau et l'année.

Une telle fréquence intensive est non seulement non rentable mais aussi dangereuse : outre les considérations individuelles, le rythme de balnéation idéal devrait permettre une infestation de tiques comprise entre la charge minimale nécessaire pour maintenir un état de stabilité enzootique vis-à-vis des maladies transmises, y compris et en premier lieu de l'ECF, et la charge maximale acceptable qui n'entraîne pas de pertes économiques notables (12). En fait, l'ignorance, à la conception de ce programme de lutte, des phénomènes de stabilité enzootique développés par les races locales de bétail a conduit celui-ci à une impasse financière et technique. Une révision est désormais nécessaire en tenant compte de l'avancée des connaissances dans l'épidémiologie des maladies à tiques ainsi que des contraintes économiques au niveau national comme du point de vue de l'éleveur.

\section{A propos d'une participation financière des éleveurs}

Le coût d'entretien et de fonctionnement du programme est tel pour les services vétérinaires que sa poursuite est sérieusement menacée, en tout cas dans sa version intensive (18). Serait-il possible que les éleveurs, qui en 
sont les principaux bénéficiaires, y contribuent financièrement si la balnéation cessait d'être obligatoire ou si son rythme était réduit ? Selon Tacher (20) une participation financière des éleveurs à un programme n'est pas envisageable s'ils n'obtiennent pas de ce programme des avantages similaires à ceux qu'ils obtiennent en utilisant leur argent dans d'autres activités. La comparaison avec l'utilisation d'engrais, courante parmi les éleveurs de la région, semble ici pertinente.

Une estimation du bénéfice net que l'éleveur tire de la balnéation peut être faite en considérant la distance médiane au bain $(4 \mathrm{~km})$, la taille médiane du troupeau ( 7,3 animaux) et une fréquence de 17 bains par an, proche de ce qui s'observe en pratique. Le bénéfice annuel net calculé est alors de 18,2 MK par troupeau.

Le bénéfice tiré de l'utilisation d'engrais, estimé lors de l'enquête, suit la relation : $(\mathrm{B}-\mathrm{Ca}) / \mathrm{Ce}=3$, avec $\mathrm{B}$ : bénéfice brut de la production agricole ; Ca : coûts hors coût des engrais ; $\mathrm{B}$ - $\mathrm{Ca}$ : bénéfice net ; $\mathrm{Ce}$ : coût des engrais. L'éleveur doit donc tirer de la balnéation un avantage similaire. Cette relation coût/bénéfice peut être appliquée comme une contrainte à la balnéation, avec B$\mathrm{C}$ : bénéfice net de la balnéation (hors taxation), et $\mathrm{Ce}$ : frais annuels de taxation, décomposables par bain (17) et par animal $(7,3)$. Le calcul aboutit à une taxe par animal et par bain qui ne peut excéder 4 tambala ; cette somme est modique en regard du coût du programme. II n'est donc pas possible d'attendre des éleveurs une contribution notable pour assurer la poursuite de la balnéation. Les résultats de 1992 (19) ont toutefois modéré cette conclusion : l'éleveur obtient un bénéfice supérieur avec un rythme de balnéation moindre, et sa contribution peut ainsi être plus élevée et atteindre 15 tambala par bain et par animal. Elle reste de toute manière insuffisante pour couvrir les coûts de fonctionnement et très insuffisante pour permettre la construction de nouveaux bains.

Il est envisagé, au niveau national, de remplacer la balnéation par des pulvérisations manuelles réalisées au site de l'ancien bain afin de réduire les coûts fixes liés à leur entretien. La situation de l'éleveur en regard des coûts et des bénéfices" n'en serait pas changée mais les frais pour les services vétérinaires seraient considérablement diminués et se rapprocheraient des 4 , et à plus forte raison des 15, tambala acceptables. Une prise en charge par les éleveurs pourrait alors s'envisager avec cette nouvelle méthode.

\section{CONCLUSION}

La lutte par bains détiqueurs présente de réels avantages pour les éleveurs mais elle n'est pas sans coût. Les diffé-

\footnotetext{
* Ceci reste une hypothèse à vérifier : l'efficacité des pulvérisations dépend du soin que l'opérateur y met ; un bain présente à cet égard plus de fiabilité.
}

rences de systèmes de production interviennent dans leur degré d'adhésion à cette technique. Les éleveurs ont par ailleurs d'autres priorités en santé animale, en conduite du troupeau ou dans d'autres domaines de leur système de production. Ces autres priorités concernant l'élevage n'ont pas reçu autant d'attention que l'ECF. Elles mériteraient d'être considérées, ce qui pourrait entraîner des améliorations rapides et peu coûteuses.

L'instauration d'une taxe à la balnéation ne représente pas une solution d'une grande portée pratique dans le cadre de la technique actuelle. Le remplacement de la balnéation par des pulvérisations manuelles est par contre une alternative a priori envisageable d'un point de vue technique et économique. Le coût de ces pulvérisations pourrait et devrait être supporté par les éleveurs bénéficiaires, ce qui permettrait de vérifier leur intérêt pour le contrôle de l'ECF. Le programme de balnéation serait à reconsidérer afin de réduire son coût de fonctionnement ; notamment, l'obligation d'un bain hebdomadaire pourrait être assouplie. Un tel changement après 60 ans de balnéation intensive nécessite un effort d'explication auprès des éleveurs à travers le réseau des assistants vétérinaires. II en est de même de toute nouvelle mesure décidée dans la future stratégie de lutte contre les tiques.

Se pose la question de savoir en définitive quelles raisons empêchent les services vétérinaires de modifier une stratégie de lutte si peu justifiée par des critères économiques. La crainte d'un mouvement de protestation violent des éleveurs est l'argument le plus souvent avancé ; or cette crainte est, comme le révèle l'analyse sociologique, sans doute surestimée.

L'absence d'avantage économique de la balnéation dans une région d'ECF enzootique incite à considérer l'installation de tels programmes en élevage traditionnel avec prudence. Le manque de rentabilité d'une balnéation intensive en élevage traditionnel en absence d'ECF a été établi en Zambie (16). Une évaluation précise de l'impact économique des maladies à tiques doit être effectuée avant toute décision, au risque d'aboutir à une situation dont les coûts et les risques deviennent difficiles à gérer. La disposition de l'éleveur à payer pour le service est une bonne indication de sa motivation réelle. La connaissance de celle-ci doit être incluse dans l'analyse du programme, non seulement pour le recouvrement des coûts mais encore comme validation de la pertinence de la lutte.

\section{Remerciements}

Cette étude s'est inscrite dans le cadre d'un projet financé par l'Overseas Development Agency. Elle a bénéficié de l'appui du Centre for Tropical Veterinary Medicine (CTVM), Edimbourg, Royaume-Uni, et du Centre national d'études agronomiques des régions chaudes (CNEARC), Montpellier, France. Les auteurs tiennent à remercier les Dr Morel (CIRAD-EMVT) et Bergès (MAC) pour leurs commentaires et avis. 


\section{F. Blanc T. Norman A. Soldan D. Chilombo M. Edelsten}

\section{Bibliographie}

1. BERGGREN S.A., 1978. Cattle ticks in Malawi. Vet. Parasitol., 4: 289297.

2. BLANC F., 1992. Farmers' attitude to dipping in Malawi. MSc dissertation, CTVM, Edinburgh, Scotland, $82 \mathrm{p}$.

3. CHRISTY P., 1986. Les tiques des bovins en Ituri (Zaïre). Epidémiologie des maladies transmises et économie de la lutte. Maisons-Alfort, France, IEMVT, $295 \mathrm{p}$

4. CREEK M., 1991. Economic evaluation of national policies for tickborne diseases and tick control. Lutte contre les tiques (phase II). Burundi, 22 p. (BDI/85/011)

5. DABORN C.J., 1981. Epidemiology of East Coast Fever in Malawi. MSc. dissertation, CTVM, Edinburgh, United Kingdom, 91 p.

6. DERVIN C., 1990. Comment interpréter les résultats d'une Analyse Factorielle des Correspondances? Paris, France, Institut Technique des Céréales et des Fourrages, $75 \mathrm{p}$.

7. ECONOMIST INTELLIGENCE UNIT, 1991. Malawi, country profile. London, United Kingdom, The Economist Intelligence Unit, $37 \mathrm{p}$.

8. GRINDLE R.J., 1981. Economic losses from East Coast Fever in Malawi. In: Advances in the control of theileriosis. The Hague, Netherlands, Martinus Nijhoff, p. 408-411.

9. KNUDSEN P.B., 1972. Animal health in the Malawi Danish cattle survey. Lilongwe, Malawi, 187 p. (Report presented to the Malawi Government)

10. LAWRENCE J.A., FOGGIN C.M., NORVAL R.A.I., 1980. The effects of war on the control of diseases of livestock in Rhodesia, Zimbabwe. Vet. Rec., 107: 82-85.

11. MOODIE P.A., 1977. Country report: Malawi. In: Henson J.B., Campbell M. eds., Theileriosis. Workshop held in Nairobi, Kenya, 7.9 December 1976. Ottawa, Canada, International Development Research Centre, p. 25 27. (Report)

BLANC F., NORMAN T., SOLDAN A., CHILOMBO D., EDELSTEN M. Farmers' attitude to dipping against East Coast Fever in Malawi. Revue Elev. Méd. vét. Pays trop., 1995, 48 (4): 307-314

Dipping against East Coast Fever (ECF) in Malawi helps reduce losses caused by the disease, which vary with dipping frequency. The only cost to the farmer is the time spent on catle handling. However, the economic impact of ECF appears limited as regards traditional farming. Farmers see ECF as secondary to other health, animal production or other farming system issues. The characteristics of these systems explain the different attitudes towards dipping among the different types of farmers. Considering the high cost of dipping, various changes in the programme have been taken into consideration.

Key words: East Coast Fever - Tick - Dipping - Mite control - Disease control - Cost - Malawi.
12. MOREL P.-C., 1987. Epidéniologie des infestatiuns du bétail par les tiques. In : Premières journées vétérinaires africaines, 31 mai-2 juin 1987 , Hammamet, Tunisie. Paris, France, OIE, p. 145-160.

13. NORMAN T.L., SOLDAN A.W., 1992. Livestock Disease Evaluation Project dipping trial, 1991 Economic Report. Lilongwe, Malawi, LDEU, $25 \mathrm{p}$

14. NXUMALO R.S., 1991. Analysis of chemical tick control in Southern Africa. MSc. dissertation, CTVM, Edinburgh, United Kingdom, 84 p.

15. PEARCE D.W., 1983. Cost-benefit analysis. London, United Kingdom, Macmillan Education Ltd., 112 p.

16. PEGRAM R.G., CHIZYITKA H.G.B., 1990. The impact of natural infestations of ticks in Zambia on the productivity of cattle and implications for tick control strategies in Central Africa. Parasitology, 32: 165 175 .

17. RAVN T.J., 1972. The economy of the survey "khola" Managers. In: The activities of the Malawi Danish cattle survey. Lilongwe, Malawi, Malawi government, 187 p. (1972 report)

18. SOLDAN A.W., NORMAN T.L., 1992. Livestock Disease Evaluation Project dipping trial. Lilongwe, Malawi, LDEU, 32 p. (1991 report)

19. SOLDAN A.W., NORMAN T.L., 1993. Livestock Disease Evaluation Project dipping trial. Lilongwe, Malawi, LDEU, 33 p. (1992 report.)

20. TACHER G., 1982. Incidences économiques de la pathologie animale tropicale. Maisonș-Alfort, France, IEMVT, 47 p.

21. WILSON S.G., 1945. Some factors affecting the incidence of East Coast Fever in Northern Province, Nyasaland. J. South Afr. vet. med. Ass., $16(2): 47-52$

22. VAN WINDEN P., BEALE G.A., 1979. Goals, motivations and practices among Northern Region smallholder cattle farmers. Lilongwe, Malawi, FAO, 45 p. (UNDP/FAO Malawi: Assistance to Livestock Development, MLW/75/020, Working Paper 26)

BLANC F., NORMAN T., SOLDAN A., CHILOMBO D., EDELSTEN M. Lucha contra la Fiebre de la Costa. Este mediante baños garrapaticidas en Malawi: el programa visto desde el punto de vista del criador. Revue Elev. Méd. vét. Pays trop., 1995, 48 (4) : 307-314

La lucha mediante baños anti garrapatas contra la Fiebre de la Costa Este (ECF) en Malawi, conduce a una reducción de las pérdidas causadas por esta enfermedad, variable según la frecuencia de los baños. El único costo para el criador es el tiempo invertido en la manipulación de los animales. Sin embargo, el impacto económico de la ECF parece limitado bajo condiciones de crianza tradicional. Los criadores lo consideran secundario con respecto a otras manipulaciones de salud o de producción animal o a otros campos del sistema dc producción. Las características de estos sistemas explican las diferencias en el interés por los baños entre los diversos tipos de criadores. Debido a los costos elevados de este método de lucha, se presentan diversas modificaciones.

Palabras clave : Fiebre de la Costa oriental - Garrapata - Inmersión Control de ácaros - Control de enfermedades - Costo - Malawi. 